\title{
Autoadjusted versus fixed CPAP for obstructive sleep apnoea: a multicentre, randomised equivalence trial
}

\author{
Konrad E Bloch, ${ }^{1,2}$ Fabienne Huber, ${ }^{1}$ Michael Furian, ${ }^{1}$ Tsogyal D Latshang, ${ }^{1}$ \\ Christian M Lo Cascio, ${ }^{1}$ Yvonne Nussbaumer-Ochsner, ${ }^{1}$ Oliver Senn, ${ }^{3}$ Erich W Russi, ${ }^{1,2}$ \\ Malcolm Kohler, ${ }^{1,2}$ Otto D Schoch, ${ }^{4}$ Alexander Turk, ${ }^{5}$ Edelbert Imhof, ${ }^{6}$ Irène Laube, ${ }^{6}$ \\ Felix Matthews, ${ }^{1}$ Robert Thurnheer ${ }^{7}$
}

\begin{abstract}
- Additional material is published online only. To view please visit the journal online (http://dx.doi.org/10.1136/ thoraxjnl-2016-209699).
\end{abstract}

${ }^{1}$ Sleep Disorders Center and Pulmonary Division, University Hospital Zurich, Zurich,

Switzerland

${ }^{2}$ Zurich Center for

Interdisciplinary Sleep Research, University of Zurich, Zurich, Switzerland

${ }^{3}$ Institute of Primary Care, University Hospital Zurich, University of Zurich, Zurich, Switzerland

${ }^{4}$ Sleep Disorders Center and Pulmonary Division, Cantonal Hospital St. Gallen, St. Gallen, Switzerland

${ }_{5}^{5}$ Sleep Disorders Center and Pulmonary Division, Zurich Rehabilitation Clinic, Wald Switzerland

${ }^{6}$ Sleep Disorders Center and Pulmonary Division, City Hospital Triemli, Zurich, Switzerland

${ }^{7}$ Sleep Disorders Center and Pulmonary Division, Cantonal Hospital Munsterlingen, Munsterlingen, Switzerland

Correspondence to Professor Konrad E Bloch, Pulmonary Division and Sleep Disorders Center, University Hospital Zurich, Rämistrasse 100, Zurich CH-8091,

Switzerland; konrad.bloch@ usz.ch

Received 8 November 2016 Revised 13 August 2017 Accepted 29 August 2017 Published Online First 5 October 2017

\section{CrossMark}

To cite: Bloch KE, Huber F, Furian $\mathrm{M}$, et al. Thorax

2018:73:174-184.

\begin{abstract}
Background The obstructive sleep apnoea syndrome (OSAS) is conventionally treated by continuous positive airway pressure set at a fixed level (fCPAP). Automatic mask pressure adjustment (autoCPAP) is increasingly used during home therapy. We investigated whether autoCPAP is equivalent to fCPAP in improving sleepiness in patients with OSAS in the long-term.

Methods In this multicentre equivalence trial, 208 patients with OSAS, with median Epworth sleepiness score (ESS) 13, apnoea/hypopnoea index 48.4/hour, were randomised to treatment with autoCPAP (5-15 mbar) or fCPAP (pressure set at the 90th percentile applied by autoCPAP during 2-4 weeks adaptation). Coprimary outcomes were changes in subjective and objective sleepiness from baseline to 2 years after treatment. Equivalence ranges were \pm 2 points in ESS and \pm 3 min sleep resistance time evaluated by recording responses to light signals.
\end{abstract}

Results At 2 years, in the intention to treat analysis, the reduction in sleepiness versus pretreatment baseline was similar in patients using autoCPAP $(n=113$, mean ESS-change $-6.3,95 \% \mathrm{Cl}-7.1$ to -5.5 ; sleep resistance time $+8.3 \mathrm{~min},+6.9$ to +9.7$)$ and fCPAP $(n=95$, mean ESS-change $-6.2,95 \% \mathrm{Cl}-7.0$ to -5.3 ; sleep resistance time $+6.3 \mathrm{~min},+4.7$ to +7.8 ). The $95 \% \mathrm{Cl}$ of difference in ESS-reduction between autoCPAP and fCPAP was -0.9 to +1.4 and the $95 \% \mathrm{Cl}$ of difference in increase in sleep resistance time was -2.6 to $+1.0 \mathrm{~min}$. Blood pressure reduction and OSAS-related costs were similar between groups.

Conclusions AutoCPAP and fCPAP are equivalent within prespecified ranges in improving subjective and objective sleepiness in patients with OSAS over the course of 2 years. Costs of these treatments are similar. Trial registration number ClinicalTrials.gov NCT00280800.

\section{INTRODUCTION}

The obstructive sleep apnoea syndrome (OSAS) is caused by intermittent upper airway collapse during sleep leading to apnoeas and hypopnoeas with hypoxemia and sleep disruption. ${ }^{1}$ Patients suffer from excessive sleepiness, impaired cognitive performance and reduced quality of life. OSAS is associated with an increased risk of traffic accidents and cardiovascular diseases. ${ }^{2}$ Epidemiological studies indicate an OSAS prevalence of 5\%-15\% in adults. ${ }^{3}$ The standard treatment is nocturnal

\section{Key messages}

What is the key question?

- Is CPAP therapy with continuous automatic mask pressure adjustment (autoCPAP) equivalent to conventional CPAP therapy with fixed mask pressure (fCPAP) in improving sleepiness in patients with obstructive sleep apnoea syndrome (OSAS) in the long term?

What is the bottom line?

- This randomised trial shows that autoCPAP and fCPAP are equivalent within prespecified ranges in improving subjective and objective sleepiness and several other outcomes in patients with OSAS over the course of 2 years while costs of these treatments are similar.

Why read on?

- The results of this study suggest that both autoCPAP and fCPAP may serve as a first-line treatment of patients with OSAS.

application of CPAP via a mask. Traditionally, the fixed level of CPAP (fCPAP) set in the device for long-term therapy is determined by manual titration in the sleep laboratory so that all apnoeas and hypopnoeas during the different sleep stages and body positions are eliminated. ${ }^{4}$ More recently, computer-controlled CPAP devices adjusting mask pressure continuously by feed-back control (autoCPAP) are increasingly used to determine the effective CPAP. ${ }^{5}$ In this approach, the therapeutic fCPAP level for home therapy is set at the 90th or 95th pressure percentile applied by an autoCPAP device during home titration over a few nights. ${ }^{6}$ The therapeutic fCPAP determined in this way agrees well with values obtained by labour-intensive manual titration. ${ }^{6}$ Some clinicians prescribe autoCPAP instead of fCPAP for long-term OSAS treatment, reasoning that the pressure required to splint the airway varies with changes in sleep stages and body position and over several nights with changes in body weight and other factors. ${ }^{7-9}$ AutoCPAP may therefore have the potential to be more effective and convenient than fCPAP by better matching the varying needs of the patient and by improving comfort through applying only the minimally required pressure. However, there is no robust evidence from randomised, 
comprehensive, long-term trials to support this assumption. The current trial evaluates the hypothesis that autoCPAP is equivalent to fCPAP in improving major manifestations of OSAS, in particular subjective and objective sleepiness and several other outcomes over a duration of 2 years. Since the selection of treatment modality may have economic consequences, healthcare costs were also assessed.

\section{METHODS}

\section{Study design and objectives}

This multicentre, randomised, parallel-group trial evaluated equivalence of autoCPAP versus fCPAP in improving subjective and objective sleepiness and other outcomes in the home therapy of patients with OSAS over a 2-year period. The trial was conducted from 1 January 2006 to 31 March 2014, at the University and Triemli Hospitals Zurich, the Cantonal Hospitals Munsterlingen and St. Gallen and the Clinic Wald, Switzerland. The protocol was approved by local ethics committees and registered at ClinicalTrials.gov (NCT00280800). Participants provided informed written consent.

Additional details on methods are provided in an online supplementary file 1 .

\section{Participants}

Patients with OSAS, aged 18-75 years, both sexes, diagnosed by a compatible history with complaints of excessive sleepiness (Epworth sleepiness score (ESS) $\geq 8)^{10}$ and apnoea/hypopnoea index $(A H I) \geq 10 /$ hour in a sleep study (see online supplementary appendix $)^{11}$ were invited to participate if they had successfully completed a 2-4 week CPAP adaptation period (see below).

\section{Interventions}

Participants were instructed in the use of CPAP and a mask was fitted. Patients then used autoCPAP (pressure 5-15 mbar) at home during a 2-4-week adaptation period. Participants using autoCPAP during adaptation for $\geq 2$ hours/night and wishing to continue CPAP therapy were randomised to subsequent treatment with either autoCPAP (pressure 5-15 mbar) or fCPAP with pressure set at the 90 th percentile applied by the autoCPAP device during adaptation. ${ }^{5}$ Either a Philips Respironics RemStar or a ResMed AutoSet device were provided according to randomisation. Follow-up visits were scheduled at 1, 3, 12 and 24 months. Patients were encouraged to contact study personnel if they had problems at any time during the study.

\section{Assessments}

A history and clinical examination were obtained. Subjective sleepiness was evaluated by the Epworth sleepiness scale, an 8 -item questionnaire with scores ranging from 0 to 24 with increasing sleepiness. ${ }^{10}$ Quality of life was evaluated by the short form medical outcome questionnaire (SF-36) ${ }^{12}$ and the functional outcome of sleep questionnaire (FOSQ) including five items reflecting aspects of sleep-related quality of life (scale 0 to 20 with increasing quality). ${ }^{13}$ The patient's health status preference was assessed by the short form six dimensions instrument (SF-6D) that provides an indirect utility index anchored at 1, perfect health and 0 , death, based on responses to nine questions comprised in the SF-36. ${ }^{14}$

Sleepiness was assessed objectively by measuring the ability to resist sleep in a non-stimulatory environment using the Oxford sleep resistance test (OSLER). ${ }^{15}$ Patients underwent four 40-min test sessions over the course of 1 day sitting in a quiet room. They had to respond to light signals appearing every $3 \mathrm{~s}$ by touching a button. Sleep resistance time was recorded as the time to seven successive missed responses (or $40 \mathrm{~min}$ if this end-of-test criterion was not met). The total number of missed stimuli was also recorded. Mean results for the four trials of 1 day are reported.

Sleep studies performed at 3,12 and 24 months in patients using CPAP included pulse oximetry, mask pressure, chest wall excursions, ECG and body position.

Ambulatory 24 hours blood pressure recordings were obtained at 30-min intervals by an automatic device with an upper arm cuff (TM2430, A\&D, Tokyo, Japan). ${ }^{16}$ High sensitivity C reactive protein and total cholesterol/high-density-lipoprotein-cholesterol ratio were determined in venous blood. ${ }^{17} 18$

Treatment adherence and the AHI during home therapy were derived from the CPAP device memory. The patient's subjective assessment of treatment effectiveness and of side effects were evaluated by questionnaires (see online supplementary appendix).

Healthcare costs were obtained from a third party perspective by collecting health insurance, physician's office and hospital bills. In addition, patients recorded expenses and hours of absence from work for health reasons.

\section{Primary outcomes and sample size}

Coprimary outcomes were the change in ESS and the change in mean sleep resistance time (OSLER) at 24 months compared with pretreatment baseline. Equivalence ranges were assumed as \pm 2 points for the Epworth score (ie, a difference of \pm 2 in the change in score by fCPAP minus change by autoCPAP) and at \pm 3 min for sleep resistance time (ie, a difference of \pm 3 in change by fCPAP minus change by autoCPAP) according to previous studies. ${ }^{19} 20$ Sample size calculations performed with estimated SD of between group difference in Epworth score of 4 and in sleep resistance time of $7 \mathrm{~min}$ based on previous trials ${ }^{1920}$ indicated that 188 participants were required, in an equivalence design using two one-sided tests, to achieve $80 \%$ power at a $5.0 \%$ significance level with equivalence limits of sleep resistance time of $\pm 3 \mathrm{~min} .{ }^{21}$ Accounting for dropouts, we aimed for a minimal number of 205 participants.

\section{Randomisation and masking}

Randomisation of participants was performed according to a 1:1 balanced block design by the study centre (Zurich University Hospital). To this end, envelopes containing codes for the treatment mode and the CPAP brand for 12-24 participants were sent to participating centres as needed. The local coordinator drew a paper with the codes for each participant. Minimisation was intended in regard to the treatment mode (autoCPAP and fCPAP) and CPAP device brand (ResMed and Philips Respironics). Participants were informed that the study purpose was to investigate effectiveness of different CPAP operating modes without giving further details. True blinding of participants and clinical caregivers was not feasible since all participants had an initial phase of autoCPAP therapy. However, data were analysed blinded to the treatment mode.

\section{Statistics}

Analyses were performed both according to the intention-totreat principle including the full analysis set and per protocol including only data from participants adhering to the protocol and using CPAP for a mean of $\geq 2$ hours/night. ${ }^{22}$ For the intention-to-treat analyses, missing data were replaced by multiple (20) imputations using regression models with chained equations. ${ }^{23}$ To this end, anthropometrics, variables from sleep 
studies, vigilance tests, blood pressure, laboratory parameters and CPAP mask pressure were entered into the models. Mean values (with SE) of outcomes at successive follow-up times and changes versus baseline (with 95\% CIs) were computed for patients treated with fCPAP and autoCPAP using multilevel mixed-effects linear regression models with time and treatment mode as predictors in unadjusted analyses. To control for potential confounders adjusted analyses were also performed with baseline values of the dependent variable, age, sex, the baseline oxygen desaturation index (as a measure of sleep apnoea severity) and the CPAP device brand as predictors. Effect sizes were computed as the mean change in any variable divided by the SD at baseline with effect sizes $>0.8$ considered as large. ${ }^{24}$ Multilevel mixed effects regression models were also employed to evaluate effects of OSAS severity and of time effects over the course of the study (2006-2014). Equivalence of autoCPAP versus fCPAP was evaluated by computing means and two-sided 95\% CIs of unadjusted and of adjusted differences in treatment effects in the two groups. ${ }^{22} 25$ A probability of $\mathrm{p}<0.05$ was assumed as statistically significant.

\section{RESULTS}

Figure 1 shows the patient flow. Of 952 patients assessed for eligibility, 208 underwent randomisation, 172 patients completed the study, 92 randomised to autoCPAP, 80 randomised to fCPAP ( $p=0.596, \chi^{2}$ test for proportions); the proportion of patients who discontinued participation because of a lack of subjective benefit was similar in the two treatment arms $\left(p=0.319, \chi^{2}\right.$ test for proportions). Participant characteristics are listed in table 1.

Tables 2 and 3 summarise the results of assessments at baseline and at 24 months along with the changes in outcomes for patients treated with autoCPAP and fCPAP. The unadjusted and the adjusted mean between group differences in changes with 95\% CIs for the intention-to-treat (table 2) and the per-protocol analyses (table 3 ) are also presented. Details of the regression

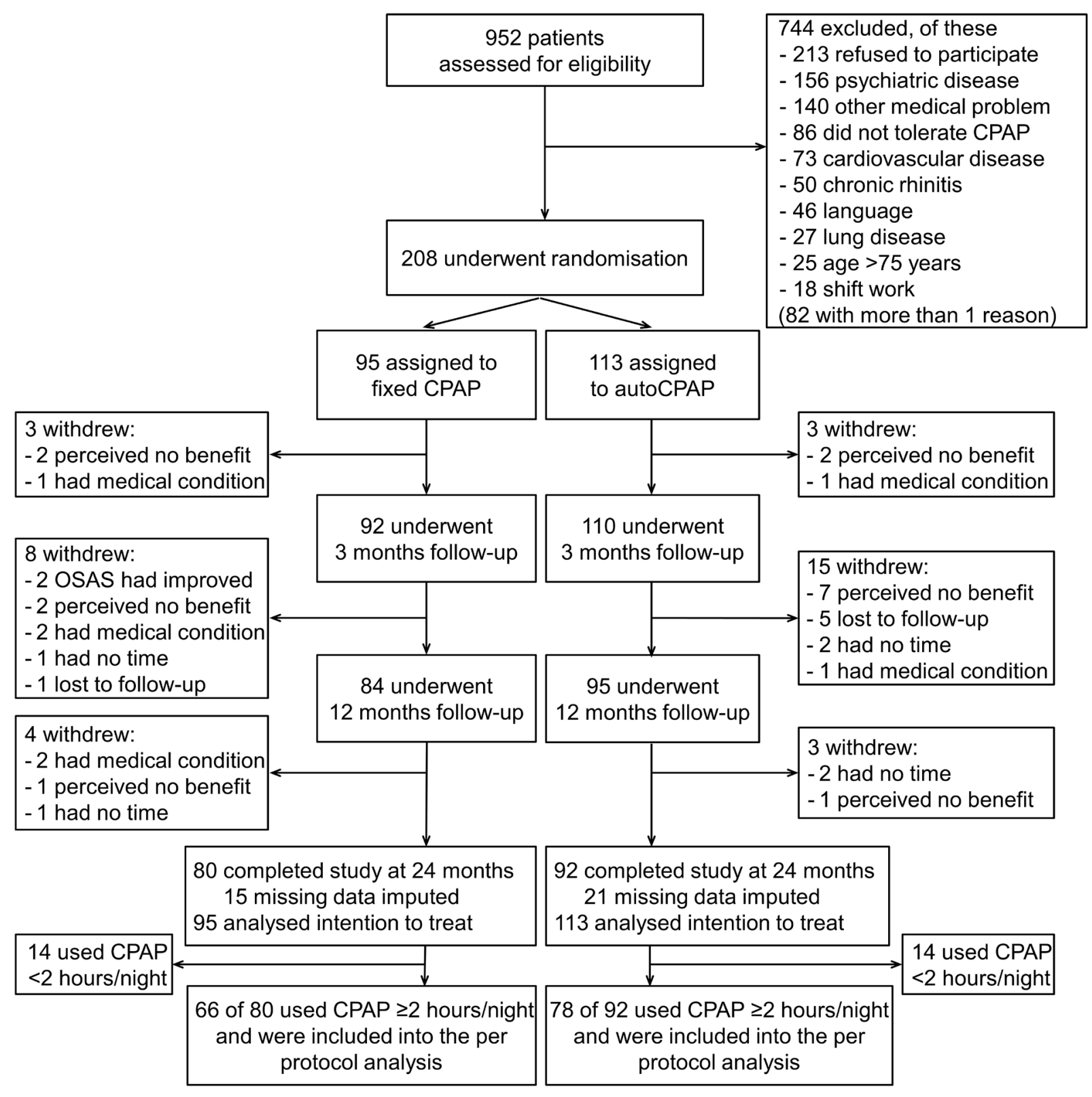

Figure 1 Patient flow. autoCPAP, CPAP with autoadjusted mask pressure; OSAS, obstructive sleep apnoea syndrome. 
Table 1 Baseline characteristics of the study participants

\begin{tabular}{|c|c|c|c|}
\hline Characteristic & All $(n=208)$ & fCPAP $(n=95)$ & autoCPAP $(n=113)$ \\
\hline Age (years) & $55.5(47.0$ to 62.0$)$ & $56.0(47.0$ to 61.0$)$ & $55.0(47.0$ to 62.0$)$ \\
\hline Male sex-no. (\%) & $180(87 \%)$ & $83(87 \%)$ & $97(86 \%)$ \\
\hline Body mass index $\left(\mathrm{kg} / \mathrm{m}^{2}\right)$ & $32.7(29.3$ to 36.9$)$ & 32.8 (29.4 to 36.9$)$ & $32.5(29.1$ to 36.8$)$ \\
\hline AHI (events/hour) & $48.4(31.4$ to 69.0$)$ & $52.2(32.1$ to 71.0$)$ & $43.0(30.0$ to 65.9$)$ \\
\hline ODI>3\% (events/hour) & $42.3(29.9$ to 61.0$)$ & $44.0(30.0$ to 65.7$)$ & $40.1(29.4$ to 57.0$)$ \\
\hline \multicolumn{4}{|l|}{ Patients—no. (\%) with ODI>3\% } \\
\hline$\leq 15$ (events/hour) & $16(8 \%)$ & $4(4 \%)$ & $12(11 \%)$ \\
\hline$>15-30$ (events/hour) & $41(20 \%)$ & $21(22 \%)$ & $20(18 \%)$ \\
\hline$>30-50$ (events/hour) & $73(35 \%)$ & $30(32 \%)$ & $43(38 \%)$ \\
\hline$>50$ (events/hour) & $78(38 \%)$ & $40(42 \%)$ & $38(34 \%)$ \\
\hline Nocturnal mean $\mathrm{SpO}_{2}(\%)$ & 93 (90 to 94$)$ & 93 (90 to 94$)$ & 92 (91 to 94$)$ \\
\hline Score on Epworth Sleepiness Scale & $13(11$ to 16$)$ & $13(11$ to 16$)$ & $13(11$ to 16$)$ \\
\hline 24 hours mean blood pressure $(\mathrm{mm} \mathrm{Hg})$ & $95(90$ to 100$)$ & $94(89$ to 100$)$ & 95 (91 to 101$)$ \\
\hline \multicolumn{4}{|l|}{ Medication use-no. (\%) } \\
\hline Statin & $24(12 \%)$ & $12(13 \%)$ & $12(11 \%)$ \\
\hline Antihypertensive medication & $148(71 \%)$ & $62(65 \%)$ & $86(76 \%)$ \\
\hline Antidiabetic medication & $10(5 \%)$ & $6(6 \%)$ & $4(4 \%)$ \\
\hline Platelet aggregation inhibitor & $26(13 \%)$ & $12(13 \%)$ & $14(12 \%)$ \\
\hline Antidepressant & $14(7 \%)$ & $8(8 \%)$ & $6(5 \%)$ \\
\hline
\end{tabular}

Values are medians (quartiles).

AHI, apnoea/hypopnoea index; fCPAP and autoCPAP, CPAP with fixed and autoadjusted mask pressure; ODI, oxygen desaturation index; $\mathrm{SpO}_{2}$, oxygen saturation by pulse oximetry.

analyses are shown in online supplementary tables 1 and 2 . Figure 2 and online supplementary figure 1 illustrate the equivalence analyses for the main outcomes.

According to the intention-to-treat analysis, patients in the autoCPAP and fCPAP group experienced significant improvements in sleepiness as reflected in reductions in the ESS to the normal range, that is, an unadjusted mean reduction of 6.3 with autoCPAP (effect size $-1.4,95 \% \mathrm{CI}-1.2$ to -1.6 ) and 6.2 points with fCPAP (effect size $-1.5,95 \% \mathrm{CI}-1.3$ to -1.7 ) at 24 months. The mean sleep resistance time increased to $39.2 \mathrm{~min}$ in the 24 months follow-up evaluations corresponding to a mean unadjusted increase of $8.3 \mathrm{~min}$ (autoCPAP, effect size 1.1, $95 \%$ CI 0.9 to 1.3 ) and $6.3 \mathrm{~min}$ (fCPAP, effect size $0.8,95 \% \mathrm{CI}$ 0.6 to 1.0$)$. Generic and sleep-related quality of life were both improved and patients preferred their health state during CPAP therapy compared with pretreatment baseline as indicated by the higher SF-6D utility index (tables 2 and 3). Sleep studies at 24 months revealed a normalisation of the AHI and the oxygen desaturation index in both groups (tables 2 and 3). Improvement in sleep apnoea was associated with a reduction in the 24 hours systolic, diastolic and mean blood pressure by $3-4 \mathrm{~mm} \mathrm{Hg}$ in the intention to treat analysis and by $4-6 \mathrm{~mm} \mathrm{Hg}$ in the per protocol analysis. A separate analysis of diurnal and nocturnal blood pressure revealed reductions by $3-5 \mathrm{~mm} \mathrm{Hg}$ (intention to treat) and by $5-9 \mathrm{mmHg}$ (per protocol), similarly in the autoCPAP and fCPAP group (see online supplementary tables 3 and 4). There were no significant changes in high sensitivity $\mathrm{C}$ reactive protein and in total cholesterol/high-density-lipoprotein-cholesterol ratio in both groups (tables 2 and 3). The mean mask pressure applied by autoCPAP was significantly lower by approximately 2 mbar than the pressure with fCPAP. The mean CPAP use was similarly high in both groups ( $>5$ hour/night at all follow-up times).
Equivalence of autoCPAP versus fCPAP was assessed by mean differences and two-sided 95\% CI of changes in outcomes at 24 months (fCPAP-autoCPAP, tables 2 and 3, figure 2 and see online supplementary figure 1$)$. In the intention-to-treat analysis, the 95\% CI of unadjusted differences in treatment effects (intentionto-treat analysis) extended from -0.9 to +1.4 points for the Epworth score and from -2.6 to $+1.0 \mathrm{~min}$ for the sleep resistance time, were well within the equivalence ranges (figure 2). The 95\% CI of differences in treatment effects are also shown for quality of life, AHI, nocturnal oxygen saturation and blood pressure (figure 2). As the CI were overlapping zero, no superiority or inferiority of any of the treatment modes was suggested. The unadjusted mean differences in outcomes between treatment modes with the 95\% CIs in the per protocol analysis corroborated the corresponding results of the intention-to treat-analysis. Moreover, the adjusted intention-to-treat and per-protocol analyses provided similar results as the corresponding unadjusted analyses with slightly reduced equivalence ranges (tables 2 and 3 , figure 2 and see online supplementary figure 1 ).

The patients perceived the treatment subjectively as very effective (see online supplementary table 5). Minor side effects such as mask leaks, dryness of the mouth or nasal obstruction were common and occurred with similar frequency with both treatment modes (see online supplementary table 5).

Regression analyses did not indicate any effect of OSAS severity quantified by the baseline oxygen desaturation index on the relative effectiveness of autoCPAP versus fCPAP in improving sleepiness in terms of the Epworth score and the sleep resistance time (see online supplementary tables 6 and 7) and there was no effect of the elapsed time during the study period 2006-2014 on the relative effectiveness of treatment modes on the Epworth score and sleep resistance time (see online supplementary tables 8 and 9). 












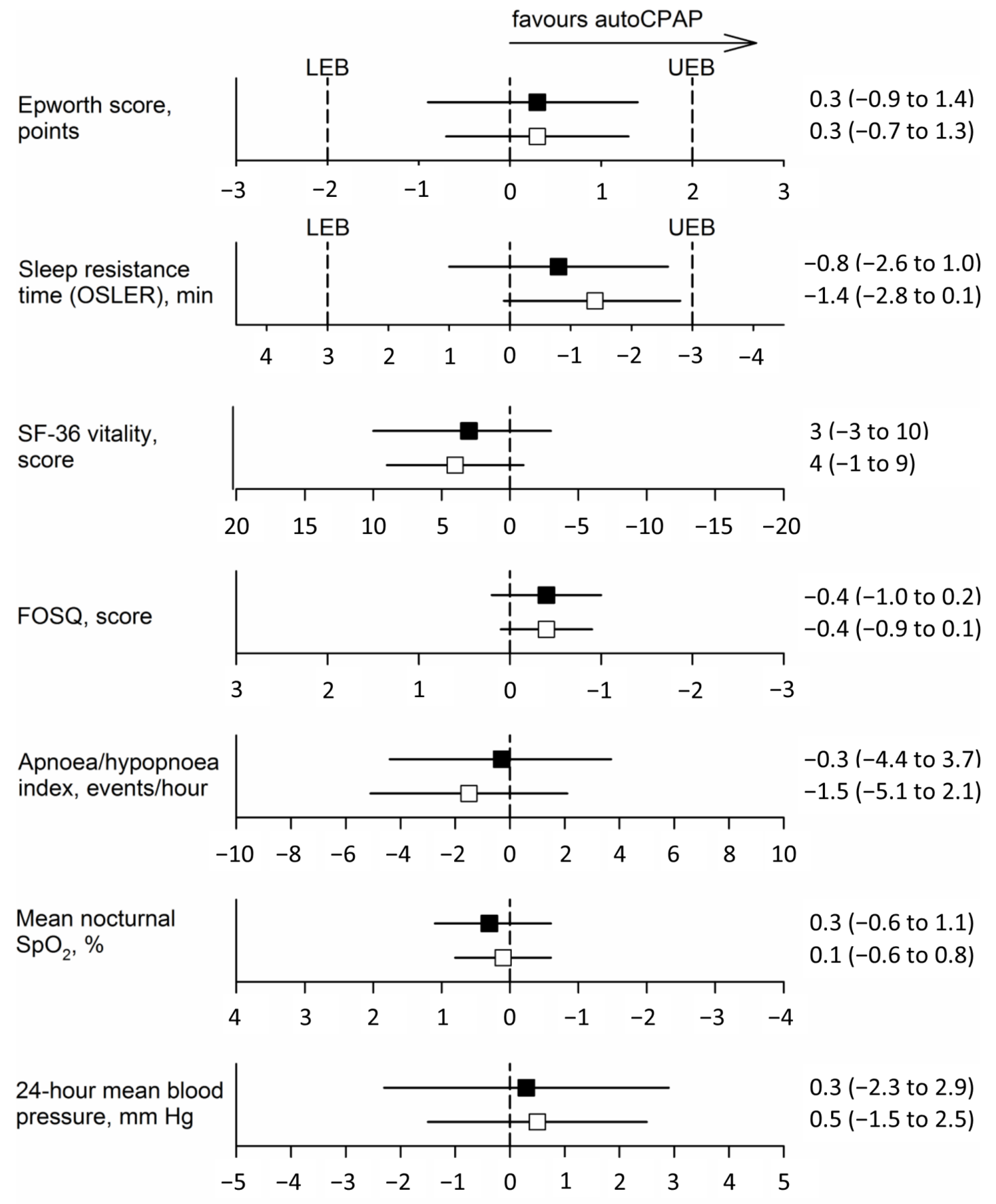

\section{Difference in changes from pretreatment baseline (fCPAP-autoCPAP) at 24 months}

Figure 2 Equivalence plots for the intention-to-treat analysis. The squares and horizontal lines represent the mean difference in changes of outcomes at 24 months versus pretreatment baseline (fCPAP minus autoCPAP) and the corresponding 95\% Cls for the unadjusted (filled symbols) and adjusted analysis (open symbols). As a favourable effect for certain outcomes is a decrease and for others it is an increase, the direction of the $x$-axes scale has been arranged so that differences in favour of autoCPAP are displayed to the right of the 0 difference. LEB and UEB for the Epworth score and the sleep resistance time, respectively, are shown as vertical dashed lines. fCPAP and autoCPAP, CPAP with fixed and autoadjusted mask pressure; FOSQ, functional outcome of sleep questionnaire; LEB, the lower boundary of the prespecified equivalence ranges; OSLER, Oxford sleep resistance test; SF-36, short form of the medical outcome questionnaire; $\mathrm{SpO}_{2}$, oxygen saturation by pulse oximetry; UEB, the upper boundary of the prespecified equivalence ranges.

The effect of CPAP therapy was most pronounced within the first 3 months but slight further changes were observed over the course of the subsequent study period up to 24 months (figure 3 and online supplementary table 10). Over the course of the 2-year study period, the cumulative OSAS-related and total healthcare costs and the hours of health-related absence from work were similar in patients treated with autoCPAP and fCPAP (table 4).

Costs for unscheduled consultations were also similar in both groups. The main costs (90\% autoCPAP group, 93\% fCPAP 

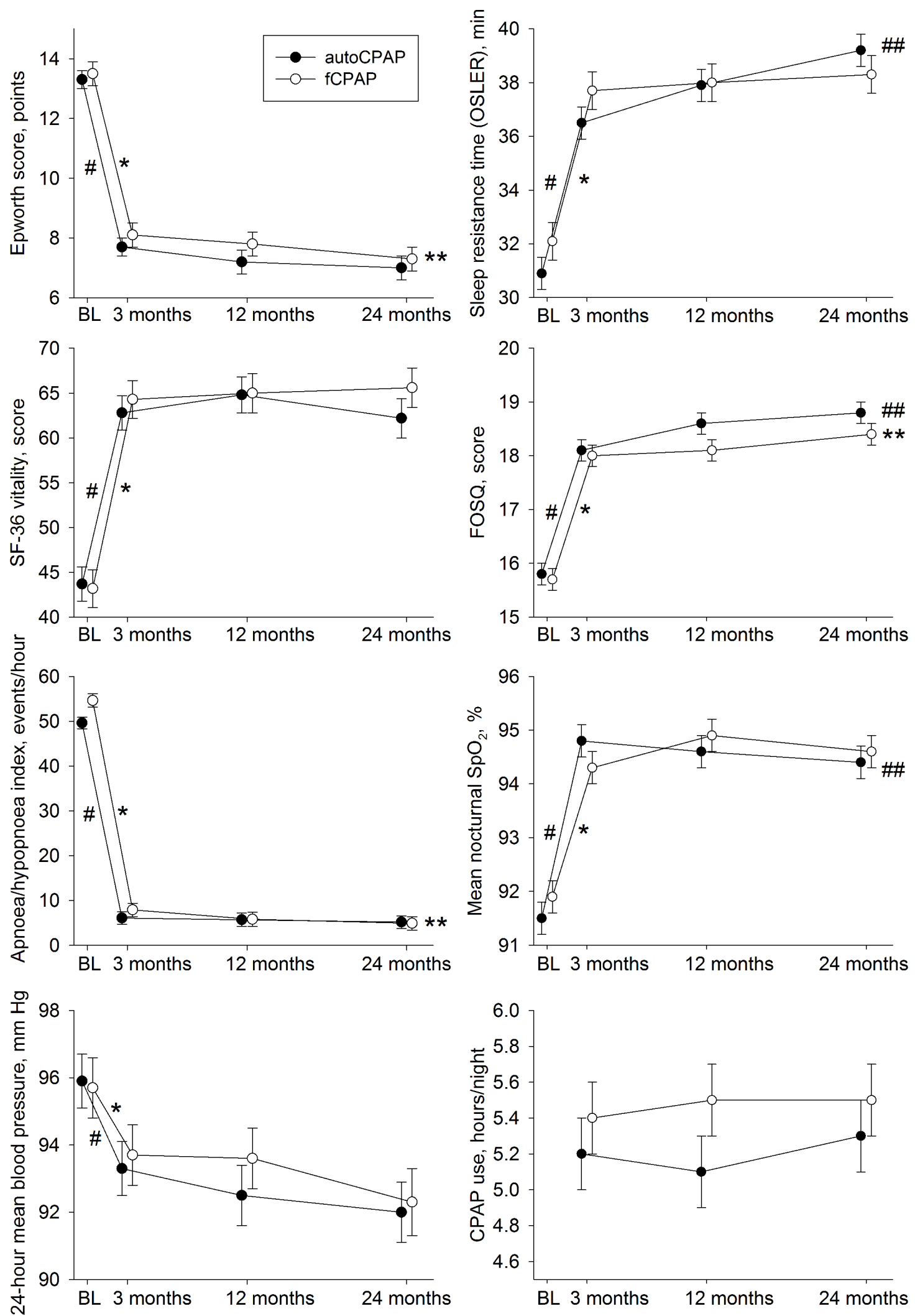

Figure 3 Changes in main outcomes over the course of the 2-year study period. The mean \pm SE of outcomes at pretreatment baseline and after 3,12 and 24 months of treatment are shown for the intention-to-treat analysis of data from patients using autoCPAP ( $n=113$, closed circles) and fCPAP ( $n=95$, open circles). Major changes occurred in the first 3 months but regression analysis of data from 3 to 24 months revealed additional minor changes in some variables (see also online supplementary table 10). * and \# indicate significant $(p<0.05)$ changes from BL to 3 months in the fCPAP and autoCPAP group; ${ }^{*}$ and \#\# indicate significant changes from 3 to 24 month in the fCPAP and autoCPAP group. BL, baseline; fCPAP and autoCPAP, CPAP with fixed and autoadjusted mask pressure; FOSQ, functional outcome of sleep questionnaire; OSLER, Oxford sleep resistance test; $\mathrm{SF}-36$, short form of the medical outcome questionnaire; $\mathrm{SpO}_{2}$, oxygen saturation by pulse oximetry. 
Table 4 Cumulative direct and indirect healthcare costs

\begin{tabular}{|c|c|c|c|c|}
\hline \multirow[b]{2}{*}{ Follow-up } & \multicolumn{2}{|l|}{ All costs } & \multicolumn{2}{|l|}{ OSAS-related costs } \\
\hline & autoCPAP & fCPAP & autoCPAP & fCPAP \\
\hline 3 months & 4.13 (3.02 to 8.90$)$ & 4.75 (2.81 to 9.84$)$ & 2.52 (1.85 to 2.95$)$ & 2.21 (1.79 to 2.98$)$ \\
\hline 12 months & $10.20(6.79$ to 18.45$)$ & 10.66 (6.35 to 20.82$)$ & 4.51 (3.73 to 5.15$)$ & 4.23 (3.71 to 4.97$)$ \\
\hline 24 months & 11.38 (7.95 to 21.19$)$ & 11.44 (7.39 to 21.18$)$ & 5.25 (4.58 to 6.11$)$ & 5.07 (4.53 to 5.74$)$ \\
\hline Unscheduled consultations* & 0.00 (0.00 to 0.48$)$ & 0.18 (0.00 to 0.43$)$ & NA & NA \\
\hline \multicolumn{5}{|c|}{ Health-related absence from work (hours) } \\
\hline & \multicolumn{2}{|l|}{ All } & \multicolumn{2}{|l|}{ OSAS-related } \\
\hline Follow-up & autoCPAP working & fCPAP working & autoCPAP & fCPAP \\
\hline 3 months & 23 (19 to 48$)$ & $34(19$ to 60$)$ & 17 (15 to 18$)$ & 16 (14 to 19$)$ \\
\hline 12 months & 53 (38 to 95$)$ & 60 (37 to 115$)$ & 28 (25 to 31$)$ & 27 (25 to 30$)$ \\
\hline 24 months & 68 (49 to 105$)$ & 70 (48 to 142 ) & $37(35$ to 40$)$ & 38 (35 to 42 ) \\
\hline Unscheduled consultations* & 0 (0 to 2$)$ & $1(0$ to 2$)$ & NA & NA \\
\hline
\end{tabular}

Cumulative costs were computed from the end of the CPAP adaptation period to the corresponding follow-up time. Costs for initial diagnosis are not included. Values are medians (quartiles) of individually recorded costs in thousand Swiss francs in 102 patients using autoCPAP and in 88 patients using fCPAP. Absence from work hours were computed for patients receiving salaries for work ( $n=70$ patients using autoCPAP, $n=67$ using fCPAP).

*Unscheduled consultations ( $n=89$ in the autoCPAP group and $n=104$ in fCPAP group) refers to telephone contacts or clinic visits related to 0SAS or CPAP-related problems. These costs are included in the cumulative costs.

fCPAP and autoCPAP, CPAP with fixed and autoadjusted mask pressure; OSAS, obstructive sleep apnoea syndrome; $p$, NS, all comparisons between autoCPAP and fCPAP at corresponding follow-up times.

group) and most of the health-related absence from work $(78 \%$ autoCPAP group, $86 \%$ fCPAP group) were observed in the first year of treatment.

\section{DISCUSSION}

The current randomised, multicentre trial in patients with OSAS shows that treatment with autoCPAP is equivalent to treatment with fCPAP in improving subjective and objective sleepiness over a 2 -year period. The $95 \% \mathrm{CI}$ of differences in treatment effects by autoCPAP and fCPAP were well within the predefined equivalence boundaries of \pm 2 points in the Epworth score and $\pm 3 \mathrm{~min}$ in sleep resistance time (figure 2 and see online supplementary figure 1). Moreover, autoCPAP and fCPAP provided similar improvements in quality of life, sleep-related breathing disturbances, blood pressure and several other health-related outcomes within narrow confidence limits. Cumulative healthcare costs over the course of the first 2 years of autoCPAP and fCPAP treatment were similar. These results support the use of autoCPAP as a convenient alternative to fCPAP in the long-term treatment of OSAS.

The strengths of the current study are its long observation period of 2 years, the design as a equivalence trial including an appropriately large sample size and assessing a comprehensive set of health outcomes as well as costs. The effects of autoCPAP on the ESS (effect size -1.4) and on the sleep resistance time (effect size 1.1) were large. The changes in these primary outcomes (tables 2 and 3) were at least as great or exceeded those achieved previously with fCPAP in patients with severe OSAS. ${ }^{2627}$ Regression analysis did not suggest any difference in effectiveness of autoCPAP and fCPAP in symptomatic patients with different OSAS severity in terms of improving sleepiness (see online supplementary tables 6 and 7). Effectiveness of the two brands of autoCPAP devices in improving various outcomes was similar (see online supplementary tables 1 and 2). We observed a high adherence of $>5$ hours/ night to both treatment modes (table 2). Limiting the analysis to patients using CPAP for a mean of $\geq 2$ hours/night (per-protocol analysis, table 3 ) did not change the conclusions from the trial.

A Cochrane meta-analysis including data from 609 patients with OSAS revealed similar effects of autoCPAP and fCPAP on subjective sleepiness assessed by the Epworth score. ${ }^{7}$ With few exceptions, ${ }^{19}{ }^{20}$ the analysed trials were not designed to evaluate equivalence or non-inferiority of autoCPAP. In a more recent meta-analysis of different trials performed in 616 patients, autoCPAP was associated with a slightly greater reduction in the Epworth score than fCPAP (mean difference -0.48 , $95 \%$ CI -0.81 to -0.15$){ }^{8}$ This result was mainly influenced by one cross-over study in 181 patients. ${ }^{28}$ The current parallel trial in 208 participants extends previous data by the considerably longer observation period of 24 months, by demonstrating equivalence of autoCPAP versus fCPAP in improving the Epworth score within $95 \%$ CIs of -0.9 to 1.4 points (figure 2 and online supplementary figure 1) and by comparing costs of the two treatments.

In four previous shorter-term studies (1-6 months observations), improvement in objective sleepiness assessed by the OSLER did not significantly differ between patients treated with autoCPAP and fCPAP. ${ }^{19} 202829$ The current study suggests equivalence of autoCPAP versus fCPAP at 24 months within the range of $\pm 3 \mathrm{~min}(95 \% \mathrm{CI}$ of difference in treatment effects -2.6 to 1.0 min, figure 2 ).

Trials comparing quality of life in patients with OSAS treated with autoCPAP versus fCPAP were heterogeneous preventing a pooled analysis. In eight trials, there was no statistically significant difference in the measure of quality of life, one cross-over trial found a slightly better quality of life in certain domains with autoCPAP than fCPAP. ${ }^{8}$ In the current study, autoCPAP and fCPAP provided similar improvements in quality of life (tables 2 and 3 , figure 2 and online supplementary figure 1).

AutoCPAP and fCPAP were equally effective in improving sleep-related breathing disturbances within narrow limits 
(95\% CI of differences in changes of AHI -4.4 to 3.7 events/ hour and in nocturnal oxygen saturation $-0.6 \%$ to $1.1 \%$ ).

Changes in blood pressure induced by autoCPAP and fCPAP in patients with OSAS have not been extensively studied. ${ }^{28} 29$ In one trial, ${ }^{30} 161$ patients using fCPAP for 4 months had no greater reduction in office systolic blood pressure, the primary outcome, than 161 patients using autoCPAP, although a slightly greater reduction (by $1.4 \mathrm{~mm} \mathrm{Hg}$ ) in the 24 hours diastolic blood pressure by fCPAP was reported. The trial was not designed to evaluate non-inferiority or equivalence. In the current study, reductions in 24 hours systolic, diastolic and mean blood pressure at 24 months were considerable with both CPAP modes (3-4 $\mathrm{mm} \mathrm{Hg}$ in the intention-to-treat analysis and $4-6 \mathrm{~mm} \mathrm{Hg}$ in the per-protocol analysis, tables 2 and 3). AutoCPAP was equivalent to fCPAP in reducing 24 hours systolic, diastolic and mean blood pressure within a $95 \%$ CIs of -2 to $3 \mathrm{~mm} \mathrm{Hg}$ (figure 2 and see online supplementary figure 1); a separate analysis of diurnal and nocturnal blood pressure confirmed equivalence of autoCPAP and fCPAP in reducing blood pressure (see online supplementary tables 3 and 4 ). The difference in cardiovascular risk associated with such a minor blood pressure difference seems small ${ }^{31}$ but regular blood pressure measurements are advisable in patients with OSAS independent of treatment.

A direct comparison of the individual subjective preference of the treatment mode as performed in some previous crossover trials was not feasible in participants of the current parallel-design trial. However, the subjectively perceived efficacy of treatment was similar in patients using autoCPAP and fCPAP (see online supplementary table 5) and the treatment adherence in the unadjusted analyses and the proportion of patients discontinuing CPAP did not differ between groups (tables 2 and 3 and figure 1). In the adjusted analyses, there was a slightly greater use of $6 \mathrm{~min} /$ night (intention-to-treat) and $24 \mathrm{~min} /$ night (per-protocol) in patients using fCPAP compared with those using autoCPAP. The clinical relevance of this difference is uncertain since the main outcomes of this trial were equivalent with the two treatment modes. The current protocol included an initial adaptation period with autoCPAP before starting longterm therapy with the assigned modality. This might have influenced the subjective perception of comfort and efficacy of the long-term therapy. However, the trial reflected the increasingly common practice of determination of effective mask pressure by autoCPAP during an adaptation period before setting a fixed CPAP for subsequent long-term treatment and the results are therefore relevant for the current clinical practice. In patients who are unable to successfully complete an adaptation period on autoCPAP additional coaching and/or consideration of other treatment options might be required.

Total and OSAS-related costs and hours of health-related absence from work in patients using autoCPAP and fCPAP were similar and dominated by costs in the first year (table 4). As the study protocol required the same procedures for initiation of CPAP treatment and follow-up in both groups, potential cost savings by using autoCPAP that does not require pressure adjustments might have been missed. A complete cost-effectiveness analysis was beyond the scope of the current study and the conclusions related to the costs of treatment cannot be extrapolated to different healthcare systems and longer time horizons. Potential limitations of our study are the comprehensive assessments during follow-up that might have influenced some of the outcomes and the relatively long recruitment period from 2006 to 2014. However, regression analysis did not suggest any change in relative effectiveness of autoCPAP and fCPAP in improving sleepiness over the study period (see online supplementary tables 8 and 9).

In conclusion, the current randomised multicentre trial demonstrates equivalence of autoCPAP and fCPAP in improving subjective and objective sleepiness over the course of 2 years in patients with different severities of OSAS and it shows similar costs associated with the two treatment modes. Taking the effectiveness and convenience of autoCPAP into account this treatment seems appropriate as a first-line therapy for OSAS.

Contributors KEB contributed to conception and design of the study, acquisition, analysis, interpretation of data and drafting the article. FH contributed to the data analysis and drafted the article with input from KEB. MF contributed to data analysis and interpretation and to the manuscript revision. RT and EWR contributed to conception and design of the study, acquisition and interpretation of data. TDL, CMLC, YN-O, OS, MK, ODS, AT, EI, IL contributed to acquisition, analysis and interpretation of data. FM contributed to study design, data acquisition and analysis. All authors reviewed and commented on the manuscript.

Funding The study was supported by the Swiss National Science Foundation, the Lung Leagues of Zurich, St. Gallen and Thurgau and by unconditional grants from the Respironics Foundation and ResMed Switzerland.

Disclaimer This was an investigator initiated trial, and the commercial companies were not involved in study design, data acquisition and analysis or writing the manuscript.

Competing interests KEB reports grants to his institution from Swiss National Science Foundation, Zurich Lung League, Respironics Foundation, ResMed Switzerland, during the conduct of the study. The other authors report no competing interests.

Patient consent Obtained.

Ethics approval Kantonale Ethikkommission Zurich.

Provenance and peer review Not commissioned; externally peer reviewed.

(c) Article author(s) (or their employer(s) unless otherwise stated in the text of the article) 2018. All rights reserved. No commercial use is permitted unless otherwise expressly granted.

\section{REFERENCES}

1 Jordan AS, McSharry DG, Malhotra A. Adult obstructive sleep apnoea. Lancet 2014;383:736-47.

2 Marin JM, Agusti A, Villar I, et al. Association between treated and untreated obstructive sleep apnea and risk of hypertension. JAMA 2012;307:2169-76.

3 Heinzer R, Vat S, Marques-Vidal P, et al. Prevalence of sleep-disordered breathing in the general population: the HypnoLaus study. Lancet Respir Med 2015;3:310-8.

4 Epstein LJ, Kristo D, Strollo PJ, et al. Clinical guideline for the evaluation, management and long-term care of obstructive sleep apnea in adults. J Clin Sleep Med 2009;5:263-76.

5 Morgenthaler TI, Aurora RN, Brown T, et al. Practice parameters for the use of autotitrating continuous positive airway pressure devices for titrating pressures and treating adult patients with obstructive sleep apnea syndrome: an update for 2007. An American Academy of Sleep Medicine report. Sleep 2008;31:141-7.

6 Masa JF, Jiménez A, Durán J, et al. Alternative methods of titrating continuous positive airway pressure: a large multicenter study. Am J Respir Crit Care Med 2004;170:1218-24.

7 Smith I, Lasserson TJ. Pressure modification for improving usage of continuous positive airway pressure machines in adults with obstructive sleep apnoea. Cochrane Database Syst Rev 2009:CD003531.

8 Ip S, D'Ambrosio C, Patel K, et al. Auto-titrating versus fixed continuous positive airway pressure for the treatment of obstructive sleep apnea: a systematic review with meta-analyses. Syst Rev 2012;1:20.

9 Xu T, Li T, Wei D, et al. Effect of automatic versus fixed continuous positive airway pressure for the treatment of obstructive sleep apnea: an up-to-date meta-analysis. Sleep Breath 2012;16:1017-26.

10 Bloch KE, Schoch OD, Zhang JN, et al. German version of the Epworth Sleepiness Scale. Respiration 1999;66:440-7.

11 Chesson AL, Berry RB, Pack A; American Academy of Sleep Medicine, American Thoracic Society, American College of Chest Physicians. Practice parameters for the use of portable monitoring devices in the investigation of suspected obstructive sleep apnea in adults. Sleep 2003;26:907-13.

12 Ware JE, Snow KK, Kosinski M, et al. SF-36 health survey: manual and interpretation guide. Boston: The Health Institute, New England Medical Center, 1993.

13 Weaver TE, Laizner AM, Evans LK, et al. An instrument to measure functional status outcomes for disorders of excessive sleepiness. Sleep 1997;20:835-43. 
14 Brazier J, Roberts J, Deverill M. The estimation of a preference-based measure of health from the SF-36. J Health Econ 2002;21:271-92.

15 Bennett LS, Stradling JR, Davies RJ. A behavioural test to assess daytime sleepiness in obstructive sleep apnoea. J Sleep Res 1997;6:142-5.

16 Parati G, Lombardi C, Hedner J, et al. Position paper on the management of patients with obstructive sleep apnea and hypertension: joint recommendations by the European Society of Hypertension, by the European Respiratory Society and by the members of European COST (COoperation in Scientific and Technological research) ACTION B26 on obstructive sleep apnea. J Hypertens 2012;30:633-46.

17 Robinson GV, Pepperell JC, Segal HC, et al. Circulating cardiovascular risk factors in obstructive sleep apnoea: data from randomised controlled trials. Thorax 2004;59:777-82.

18 Yokoe T, Minoguchi K, Matsuo $\mathrm{H}$, et al. Elevated levels of C-reactive protein and interleukin- 6 in patients with obstructive sleep apnea syndrome are decreased by nasal continuous positive airway pressure. Circulation 2003;107:1129-34.

19 Senn O, Brack T, Matthews F, et al. Randomized short-term trial of two autoCPAP devices versus fixed continuous positive airway pressure for the treatment of sleep apnea. Am J Respir Crit Care Med 2003;168:1506-11.

20 Nussbaumer Y, Bloch KE, Genser T, et al. Equivalence of autoadjusted and constant continuous positive airway pressure in home treatment of sleep apnea. Chest 2006;129:638-43.

21 Julious SA. Sample sizes for clinical trials with normal data. Stat Med 2004;23:1921-86.

22 Piaggio G, Elbourne DR, Pocock SJ, et al. Reporting of noninferiority and equivalence randomized trials: extension of the CONSORT 2010 statement. JAMA 2012;308:2594-604.

23 White IR, Royston P, Wood AM. Multiple imputation using chained equations: Issues and guidance for practice. Stat Med 2011;30:377-99.
24 Kazis LE, Anderson JJ, Meenan RF. Effect sizes for interpreting changes in health status. Med Care 1989;27:S178-89.

25 Jones B, Jarvis P, Lewis JA, et al. Trials to assess equivalence: the importance of rigorous methods. BMJ 1996;313:36-9.

26 McDaid C, Griffin S, Weatherly H, et al. Continuous positive airway pressure devices for the treatment of obstructive sleep apnoea-hypopnoea syndrome: a systematic review and economic analysis. Health Technol Assess 2009;13:iii-iv, xi-xiv, 1-119, 143-274.

27 Bratton DJ, Gaisl T, Schlatzer C, et al. Comparison of the effects of continuous positive airway pressure and mandibular advancement devices on sleepiness in patients with obstructive sleep apnoea: a network meta-analysis. Lancet Respir Med 2015;3:869-78.

28 Vennelle M, White S, Riha RL, et al. Randomized controlled trial of variable-pressure versus fixed-pressure continuous positive airway pressure (CPAP) treatment for patients with obstructive sleep apnea/hypopnea syndrome (OSAHS). Sleep 2010;33:267-71.

29 West SD, Jones DR, Stradling JR. Comparison of three ways to determine and deliver pressure during nasal CPAP therapy for obstructive sleep apnoea. Thorax 2006;61:226-31.

30 Pépin JL, Tamisier R, Baguet JP, et al. Fixed-pressure CPAP versus auto-adjusting CPAP: comparison of efficacy on blood pressure in obstructive sleep apnoea, a randomised clinical trial. Thorax 2016;71:726-33.

31 Law MR, Morris JK, Wald NJ. Use of blood pressure lowering drugs in the prevention of cardiovascular disease: meta-analysis of 147 randomised trials in the context of expectations from prospective epidemiological studies. BMJ 2009;338:b1665. 